\title{
Práticas e desafios para a terapia ocupacional no contexto da intervenção precoce
}

\section{Practices and challenges for occupational therapy in the context of early childhood intervention}

\author{
Bruna Pereira Ricci Marini' ${ }^{1}$, Patrícia Carla de Souza Della Barba²
}

http://dx.doi.org/10.11606/issn.2238-6149.v31i1-3p17-23

\begin{abstract}
Marini BPR, Della Barba PCS. Práticas e desafios para a terapia ocupacional no contexto da Intervenção Precoce. Rev Ter Ocup Univ São Paulo. 2020 jan.-dez.;31(1-3):17-23.
\end{abstract}

RESUMO: Objetivos: analisar as práticas implementadas por terapeutas ocupacionais atuantes em serviços de Intervenção Precoce destinados a crianças entre zero e cinco anos e apontar os desafios enfrentados por esses profissionais durante a estruturação de suas ações. Procedimentos metodológicos: Trata-se de um estudo transversal, descritivo e exploratório, de abordagem qualitativa. Participaram quatro terapeutas ocupacionais vinculados a serviços localizados em municípios do interior e litoral do estado de São Paulo. A coleta de dados foi realizada mediante entrevistas semiestruturadas e a análise dos dados foi feita sob a perspectiva da Análise de Conteúdo, na modalidade de análise temática. Resultados: Os resultados do estudo, foram classificados em quatro núcleos temáticos, sendo eles: Práticas no contexto da Intervenção Precoce, O objeto da intervenção, Fundamentação para atuar e Desafios para a Terapia Ocupacional em Intervenção Precoce. Conclusões: Concluise que práticas desenvolvidas têm se estruturado sob referenciais predominantemente reabilitativos e pautados nas necessidades das crianças atendidas, o que impacta a identidade das ações. Assim, os principais encontram-se relacionados às características dos serviços, formação profissional e ao delineamento do um modelo de prática.

DESCRITORES: Terapia ocupacional; Intervenção precoce (Educação); Pré-escolar; Reabilitação
Marini BPR, Della Barba PCS. Practices and challenges for occupational therapy in the context of Early Childhood Intervention. Rev Ter Ocup Univ São Paulo. 2020 Jan-Dec;31(1-3):17-23.

\begin{abstract}
Objectives: to analyze the practices implemented by occupational therapists working in Early Childhood Intervention services for children between zero and five years old and to point out the challenges faced by these professionals during the structuring of their actions. Methodology: This is a cross-sectional, descriptive, and exploratory study, with a qualitative approach. Four occupational therapists associated with services participated in cities in the interior and coast of the state of São Paulo. Data collection was carried out through semi-structured interviews and data analysis was carried out from the perspective of Content Analysis, in the thematic analysis modality. Results: The results of the study were classified into four thematic groups: Practices in the context of Early Childhood Intervention, The object of the intervention, Rationale for action and Challenges for Occupational Therapy in Early Childhood Intervention. Conclusion: it is concluded that developed practices have been structured under predominantly rehabilitative references and based on the needs of the children served, which impacts de identity of the actions. Thus, the main ones are related to the characteristics of the services, professional training, and the design of a model of practice.
\end{abstract}

KEYWORDS: Occupational therapy; Early intervention (Education); Child, preschool; Rehabilitation

Parte da dissertação do Mestrado de Bruna P. R. Marini “As práticas de Intervenção Precoce no estado de São Paulo", orientação de Patrícia Carla de S. Della Barba, Programa de Pós-Graduação em Terapia Ocupacional da UFSCar. Apresentado no XV Encontro Nacional de Docentes de Terapia Ocupacional, Vitória - ES, 6-10 nov. 2016.

1. Mestre pelo Programa de Pós-Graduação em Terapia Ocupacional da Universidade Federal de São Carlos. Bolsista CAPES. https://orcid.org/0000-0002-0375-4735. Email: brunamarini_to@yahoo.com.br.

2. Professora Associada do Departamento de Terapia Ocupacional e do Programa de Pós-Graduação em Terapia Ocupacional da Universidade Federal de São Carlos. https://orcid.org/0000-0002-7893-8133. Email: patriciadellabarba@gmail.com.

Endereço para correspondência: Patrícia Carla Souza Della Braba. Departamento de Terapia Ocupacional. Rodovia Washington Luís, km 235 - SP-310 São Carlos, São Paulo. CEP: 13565-905 


\section{INTRODUÇÃO}

om o avanço dos conhecimentos científicos acerca
do impacto determinante que as experiências na primeira infância produzem sobre o desenvolvimento humano, a Intervenção Precoce (IP) passou a constituir, reconhecidamente, um campo para os profissionais que atuam com o público infantil, dentre eles os terapeutas ocupacionais $^{1-3}$.

Observa-se que, ao longo da constituição histórica dessa profissão, os atendimentos em pediatria foram moldandose à intervenção sob as condições neuropsicomotoras que pudessem, de alguma forma, interferir no desempenho das ocupações comuns à infância, dentre elas o brincar. Tal movimento, por sua vez, levou à um afunilamento do público alvo de suas ações, que se constituiu majoritariamente pelas crianças com deficiências, síndromes, transtornos globais do desenvolvimento elou de aprendizagem o que, consequentemente, contribuiu para a adoção de um enfoque eminentemente clínico-reabilitativo às práticas ${ }^{4}$.

Contudo, investigações atuais têm posto em xeque a eficácia desse modelo de atuação, demonstrando que as experiências inerentes aos contextos naturais de participação das crianças podem produzir efeitos semelhantes ou superiores aos das intervenções clínicas, tanto por sua qualidade quanto pela frequência, duração e significado ${ }^{5-7}$. Dessa forma, pontuase a transição do paradigma reabilitativo, centrado nos déficits e nos profissionais para o centrado nas capacidades, nos contextos naturais e na família, ampliando a possibilidade de atenção para além das questões relativas à saúde.

Países como Estados Unidos e Portugal têm experimentado com sucesso a implantação de programas pautados nessas premissas, conceituando a IP como um conjunto de serviços/recursos para crianças em idades precoces e suas famílias, que são disponibilizados quando são solicitados pela família, num certo período de vida da criança incluindo qualquer ação realizada quando a criança necessita de apoio especializado para assegurar e incrementar o seu desenvolvimento pessoal, fortalecer as auto competências da família e promover sua inclusão social (p.7) ${ }^{8}$.

No Brasil, no entanto, alguns desafios ainda se colocam para a implementação de práticas que incorporem tais conceitos, como a superação do modelo reabilitativo, o investimento em formação profissional e em estudos sobre a temática ${ }^{9-13}$. Especificamente no que confere aos terapeutas ocupacionais pontua-se ainda outro desafio, uma vez que, partindo-se do pressuposto de que esses fundamentam-se nas experiências cotidianas, papéis e ocupações para intervir sob o desenvolvimento infantil ${ }^{4}$, deveriam ser os profissionais a desenvolver as ações mais próximas àquelas recomendadas pela literatura atual, porém, esse ainda parece um cenário distante do encontrado.

Dessa forma, com vistas a ampliar tal discussão, o presente estudo objetivou analisar as práticas implementadas por terapeutas ocupacionais atuantes em serviços de Intervenção Precoce (IP) destinados a crianças entre zero e cinco anos e apontar os desafios enfrentados por esses profissionais durante a estruturação de suas ações.

\section{PROCEDIMENTOS METODOLÓGICOS}

Trata-se de um estudo transversal, descritivo e exploratório, de abordagem qualitativa. Participaram quatro terapeutas ocupacionais vinculados a serviços de IP destinados a crianças entre zero e cinco anos, localizados em municípios do interior e litoral do estado de São Paulo. A seleção dos serviços onde foram coletados os dados se deu de forma não probabilística e por conveniência. Para tanto, selecionouse a área de abrangência do Programa São Paulo pela Primeiríssima Infância (PSPPI), composta por 38 municípios, distribuídos em 5 regionais. Foram feitos contatos com as Secretarias Municipais de Saúde e levantamento dos Centros de Especializados em Reabilitação através do Cadastro Nacional de Estabelecimentos de Saúde, o que resultou na identificação de 10 serviços que concordaram em participar da pesquisa e, após o preenchimento do questionário de caracterização, selecionou-se um de cada regional segundo os critérios: a) presença de indicadores de práticas centradas na família; b) tempo de existência da equipe de IP, priorizando as mais antigas; c) composição da equipe, priorizando as com maior diversidade de profissionais; d) tempo de atuação dos profissionais na equipe, priorizando aquelas nas quais os profissionais atuam há mais tempo. Não foram identificados serviços de IP em uma das regionais. Os critérios para a inclusão dos participantes foram: a) possuir vínculo formal com serviço de IP e b) concordar em participar da pesquisa assinando o Termo de Consentimento Livre e Esclarecido (TCLE).

Dessa forma, participaram do estudo quatro terapeutas ocupacionais, dos quais dois estavam vinculados a serviços públicos e dois a entidades da sociedade civil. O tempo de atuação dos profissionais nesses serviços variou entre 4 meses e 22 anos.

A coleta de dados foi realizada mediante entrevista semiestruturada, a qual continha 27 questões acerca das diferentes etapas de intervenção, tais como: referência, primeiros contatos, avaliação, desenvolvimento do plano de intervenção, implementação e monitoramento, avaliação dos resultados e transição, bem como acerca da qualidade técnica 
dos profissionais, envolvendo questões sobre o referencial teórico empregado e os desafios enfrentados ao longo do processo de capacitação continuada.

As entrevistas foram realizadas presencialmente pela pesquisadora, gravadas e posteriormente transcritas. A análise dos dados foi feita sob a perspectiva da Análise de Conteúdo, na modalidade de análise temática, através da qual os discursos foram lidos, organizados e classificados em núcleos ${ }^{14}$.

Esse estudo foi submetido para apreciação pelo Comitê de Ética em Pesquisa com Seres Humanos da Universidade Federal de São Carlos, tendo sido aprovado em 11 de maio de 2016, sob o parecer de número 1.539.965.

\section{RESULTADOS}

A seguir, apresentam-se os resultados desse estudo, os quais foram classificados em quatro núcleos temáticos, sendo eles: Práticas no contexto da IP, O objeto da intervenção, Fundamentação para atuar e Desafios para a TO em IP.

\section{Práticas no contexto da IP}

Os resultados do presente estudo permitiram identificar que as práticas desenvolvidas por terapeutas ocupacionais no contexto da IP permanecem direcionadas exclusivamente à criança. Os relatos demonstram que, ao longo do processo terapêutico (avaliação, planejamento, intervenção, reavaliação e alta), as ações têm sido estruturadas com vistas exclusivamente às demandas apresentadas por essas.

"Primeiro a avaliação, primeiro a anamnese? Com os pais, depois a avaliação, depois, de acordo com a minha avaliação, vou ver quais são as demandas maiores da criança e começo a trabalhar em cima disso e faço meu planejamento". (P.T.)

“(...) Então a criança fica dois meses sendo avaliada, vem toda semana ou vem quinzenal. É uma etapa até chegar na gente”. (P.M.)

Outra importante característica observada refere-se à atuação, quase que exclusiva, em contexto institucional. As informações relativas à participação e desempenho das crianças em contextos externos ao ambiente terapêutico são adquiridas de forma indireta, com base no relato dos pais, professores ou outros profissionais que as acompanhem.

“(...) na primeira avaliação, no primeiro contato conhecemos a criança e a família, a família participa.
Conversamos sobre a criança, como é em casa, na escola, (...) e depois avaliamos a criança sem a mãe (...)”. (P.M.)

"Fomos várias vezes em domicílio, mas não para avaliação (...) avaliamos aqui e se houver necessidade a gente vai em domicilio". (P.Y)

"Na entrevista com os pais, já pergunta tudo (...) anota e em cima disso a gente vai trabalhando”. (P.T)

A participação dos pais, portanto, permanece limitada ao fornecimento de informações acerca do histórico da criança e ao aprendizado de técnicas e recebimento de orientações para a continuidade da intervenção em domicílio.

“(...) estamos sempre conversando com as mães, dando orientações, inclusive dando orientações de como que ela pode ajudar em casa, de como que ela pode lidar com a criança. Às vezes tem exercícios mais simples que os pais conseguem fazer, então a gente também mostra”. (P.T)

"fui vendo algumas dificuldades de preensão grossa dela e falei: "faz assim, faz essa massagenzinha". As vezes até na leitura eu divido,(...) Eu leio com a criança para a mãe ver (...). (P.Y.)

\section{O objeto da intervenção}

Observando, a partir dos relatos, que as avaliações utilizadas pelos terapeutas ocupacionais têm balizado os componentes alvo das intervenções que propõem, conclui-se que as ações em IP têm se concentrado em aspectos físicos, psíquicos elou sensoriais, que centralizam na deficiência o objeto de sua prática.

“(...) das questões motoras ... pensando nas questões de independência e das questões cognitivas, daquilo que ela tem mais dificuldade na questão do aprendizado". (P.I.)

“(...) observo desde estimulação sensorial, como é que está a integração sensorial da criança, e a parte ortopédica (...) como está a coordenação motora fina, faço uma avaliação completa do paciente, para definir o que trabalhar". (P.T.)

A adoção de tal, demonstra encontrar-se fortemente vinculada ao caráter reabilitativo proposto pelas instituições nas quais atuam e ao público circunscrito por elas, contexto no qual a deficiência desponta como critério de elegibilidade para a IP. 
"Em específico, para a terapia ocupacional, são os sindrômicos, as lesões neurológicas, os atrasos de desenvolvimento e os distúrbios de aprendizagem (...) das crianças que têm um comprometimento cognitivo mais grave (...) em geral, são esses os casos que chegam. (P.I.)

"aqui só são encaminhadas crianças com alguma deficiência múltipla ...Ou autismo, paralisia cerebral ou deficiência intelectual (...). (P.T.)

A esse respeito, observa-se que, a depender do caráter da instituição, nem sempre será o terapeuta ocupacional quem selecionará os pacientes que serão atendidos. Essa realidade foi descrita por alguns dos participantes e constituem um fator importante para a reflexão acerca do porque das práticas manterem-se dirigidas exclusivamente à deficiência.

“(...) aqui fazemos uma avaliação específica (...) no setor de triagem decidem que é melhor a criança passar por TO $e$ Fonoaudiólogo, que a criança não precisa de um fisioterapeuta. (...) eu como TO preparo uma avaliação específica, ... converso com a fono, trocamos informações". (P.M.)

\section{Fundamentação para atuar}

Em relação à fundamentação das práticas de IP desenvolvidas, os terapeutas ocupacionais referiram utilizar diferentes métodos e técnicas, como observado nos excertos abaixo:

“(...) no caso de transtorno de aprendizagem, ... a partir dos três (anos), ... tenho um método baseado em Terapia Ocupacional Dinâmica”. (P. Y.)

“(...) eu gosto bastante da integração sensorial (...)”. (P.T)

“... eu uso [Método] Bobath (...)”. (P.I.)

No entanto, apesar de citarem o emprego de referenciais teóricos, 3 dos 4 profissionais entrevistados não possuem a formação específica para sua aplicação, utilizando-se de conceitos básicos adquiridos por meio de leitura de conteúdos relacionados à temática ou transmitidos por outros profissionais que atuam na mesma instituição.

"temos um curso aqui na instituição... com uma, fisioterapeuta, que fez vários cursos de integração sensorial, ... não fiz outros cursos, nenhuma especialização". (P. M.) "procuro usar ... integração sensorial, só que eu não tenho curso nenhum". (P.Y.)

A partir dos relatos é possível observar ainda que, mesmo que alguns possuam formação teórico metodológica para o emprego de tais técnicas, as ações terapêuticas ocupacionais em IP têm sido construídas, em grande parte, com respaldo nas percepções subjetivas em relação às respostas apresentadas pelas crianças, o que leva ao emprego de abordagens mistas elou fundamentadas em experiências práticas anteriores.

“(...) não tem um método específico (...) integração sensorial, coordenação global, coordenação motora fina. Não tem um método específico que eu uso..." .(P.T.)

"Então eu já tive paixões ... de fazer só o Bobath, de fazer só o Kabath, de usar Piaget, Wallon, (...) mas não dá tempo, (...) resolvi juntar as coisas. Então tem uma coisa do Kabath que me serve, tem uma coisa do Brunston que me serve, o Bobath". (P.I.)

\section{Desafios para a TO em IP}

A partir dos relatos feitos pelos terapeutas ocupacionais é possível identificar desafios relacionados tanto ao cotidiano das práticas quanto à formação necessária para a atuação no contexto da IP.

Em relação às práticas propriamente ditas, os principais desafios apontados relacionam-se à sobrecarga de trabalho, que culmina em ausência de tempo para realização de planejamentos e práticas que se estendam para além do contexto da instituição; assim como ao atendimento em caráter generalista, que não permite ao profissional aprofundar seus conhecimentos e desenvolver habilidades para intervir especificamente com a população alvo da IP.

“(...) quando eu comecei, há bastante tempo atrás, isso era mais organizado. Tinha uma ficha, o que tinha no plano de trabalho, tratamento, avaliação. Tinha orientações escritas. Hoje não dá tempo de fazer isso". (P.I.)

(...) ir para outros lugares que a criança frequenta, [não posso]. acho que não daria tempo. É um atendimento seguido do outro. (...) Se eu tiver que participar, em algum momento, fora da instituição, eu tenho que cancelar tudo naquele dia pra poder participar (...) então a maior limitação mesmo é o tempo". (P.M.) 
"Agora a gente está recebendo cursos, inclusive teve um curso do AVASUS (...) eu não estou fazendo, porque eu estou fazendo pós em acupuntura (...) eu comecei, mas interrompi, porque a minha demanda agora não é tanto pediatria. Eu fui contratada para trabalhar com neuropediatria e [hoje] minha maior demanda é é acidente vascular encefálico (AVE)”. (P.Y.)

Nesse contexto, foi unânime a pontuação de que a formação continuada depende exclusivamente do interesse dos profissionais, com pouco ou nenhum auxílio das instituições, característica tal que pode interferir diretamente na qualidade do atendimento prestado, uma vez que não se garante o alinhamento com as propostas mais reconhecidas para esse cuidado.

“Então, estamos sempre lendo... por iniciativa nossa. Trocamos materiais e informação, pesquisa, mas é cada um por si”. (P.M.)

O desafio para usar um protocolo baseado em evidências ou uma abordagem nova baseada em evidência, depende só da gente. Porque, pelo serviço, ficaria como há vinte anos atrás (...) . a capacitação depende da gente”. (P.I.)

\section{DISCUSSÃO}

Os resultados deste estudo evidenciaram características relacionadas à diferentes aspectos da prática de terapeutas ocupacionais participantes no contexto de serviços de Intervenção Precoce, dentre elas o direcionamento das ações exclusivamente às necessidades das crianças atendidas, o emprego de abordagens reabilitativas com enfoque biomédico, a fragilidade no emprego dos referenciais teóricos e o impacto da precariedade da oferta de educação continuada sobre a prestação do serviço.

Tais resultados podem ser comparados aos encontrados no cenário geral da Intervenção Precoce no Brasil, como o relatado no estudo de Marini et al. ${ }^{10}$.

As práticas e os modelos de IP parecem desenvolver-se exclusivamente aliados ao setor da saúde, com forte prevalência de práticas voltadas à estimulação de habilidades, por meio do emprego de abordagens clínicas, estruturadas a partir de um modelo reabilitativo de cuidado e com enfoque centrado na criança. Tais características, associadas à notável escassez de literatura sobre a temática e à divergência conceitual existente, apontam para a necessidade de um esforço nacional para a atualização profissional e para a adoção de práticas que se aproximem daquelas recomendadas e reconhecidas como boas práticas pela literatura internacional (p.7).
Outro ponto a ser destacado é a constatação de que os profissionais utilizam algumas estratégias que poderiam ser potencializadas; entretanto, não demonstram conhecimento sobre como fazê-lo, sendo o caso das visitas domiciliares e intervenção fora do contexto da instituição de reabilitação. Nesse sentido, Della Barba ${ }^{15}$ pontua que, embora se tenha estrutura e conhecimento divulgado para inovar em determinadas estratégias (como as visitas domiciliares baseadas nas rotinas), ainda verifica-se que os profissionais não valorizam a prática das visitas como uma estratégia de intervenção, o que remete à necessidade de repensar como as equipes e os terapeutas ocupacionais têm se dirigido às famílias e quais modelos de parceria podem ser aprendidos com as respostas dadas pelas mesmas.

No que se refere à fundamentação empregada pelos terapeutas ocupacionais, os resultados evidenciam lacunas as quais encontram-se relacionadas tanto ao processo formativo quanto à seleção e aplicação de referenciais que sejam condizentes às demandas apresentadas e ao trabalho que se pretende desenvolver. Assim, verifica-se uma inconsistência em relação à identidade das ações desempenhadas no âmbito da Intervenção Precoce, sendo a idade dos pacientes o único elo em comum dentre as intervenções desenvolvidas nos serviços estudados.

A esse respeito, como pontuado por Constantinidis e Cunha ${ }^{16}$, a pluralidade teórico-prática aliada à ausência de um "repertório comum, resultado da falta de sistematização de conhecimentos das ações e da produção cientifica da profissão", têm impactado sob seu reconhecimento social, fragilizando as especificidades em um contexto de transformação dos modelos de atuação em saúde. Nesse sentido, verifica-se ainda que, apesar da implantação das Diretrizes Curriculares Nacionais para a formação em Terapia Ocupacional, a nível de graduação e pós graduação os conteúdos referentes à infância têm se pautado, em sua grande maioria, no aprendizado de técnicas e procedimentos direcionados ao tratamento de comorbidades físicas e sensoriais bastante pontuais e específicas, as quais, contudo, não têm se mostrado suficientes para suprir as demandas apresentadas pela população, exigindo do profissional a elaboração de estratégias baseadas em sua experiência prática.

Cabe ressaltar que, no âmbito desse estudo, os aspectos relativos à formação também foram apontados pelos próprios TOs enquanto desafios para a prática, sendo este cenário semelhante ao descrito por outros profissionais que atuam no contexto da IP no Brasil ${ }^{9,10,13}$. Essa característica evidencia a existência um regime de trabalho pautado em resultados quantitativos, ou seja, no atendimento do maior número de crianças possível, com pouco ou nenhum investimento na estruturação dos serviços ou em 
programas de educação continuada por parte dos gestores e financiadores ${ }^{10}$. Assim, verifica-se que tal investimento tem sido feito de maneira frágil e pessoal pelos terapeutas ocupacionais, os quais buscam capacitar-se através de leituras, da troca de experiências com outros profissionais elou pela participação em atividades formativas (cursos, especializações, oficinas) cuja seleção encontra-se, por vezes, mais relacionada às áreas de interesse do profissional do que às demandas apresentadas pela população alvo do serviço no qual atuam.

As fragilidades no processo de educação continuada parecem impactar negativamente na oferta de serviços que sobreponham o modelo multiprofissional, uma vez que predomina o emprego de condutas terapêuticas fragmentadas segundo o enfoque exclusivo de cada área de formação.

Tais evidências sugerem a necessidade de atualização dos referenciais teóricos empregados na formação dos terapeutas ocupacionais e, especificamente no que se refere à atuação em IP, o estabelecimento de diretrizes que auxiliem na uniformização dos cuidados prestados em uma lógica que supere o modelo biomédico, promovendo o enfoque em aspectos da participação em atividades, dos contextos naturais de aprendizagem e de práticas centradas não apenas nas crianças, mas também em suas famílias, em uma lógica que amplie os efeitos da intervenção e favoreça a delimitação de estratégias com vistas ao fortalecimento do campo profissional. Do mais, verificase ainda a necessidade de investimento na formação das equipes favoreceria a estruturação de serviços mais consistentes, com práticas alinhadas sob a perspectiva inter ou transdisciplinar.

\section{CONCLUSÕES}

Os resultados do presente estudo permitem concluir que as práticas desenvolvidas pelos terapeutas ocupacionais participantes no campo da Intervenção Precoce têm se estruturado sob referenciais predominantemente reabilitativos e pautados nas necessidades das crianças atendidas, especialmente em suas deficiências, o que impacta a identidade das ações desenvolvidas. Nesse sentido, pontuase que os principais desafios para uma atuação consistente se encontram relacionados às características dos serviços, formação profissional e ao delineamento de um modelo de prática que favoreça o fortalecimento das especificidades da terapia ocupacional em uma lógica inter transdisciplinar.

A esse respeito, convém destacar que o pequeno número de participantes e a área de abrangência restrita a alguns municípios do estado de São Paulo constituem limitações deste estudo, indicando a necessidade de ampliação e aprofundamento na investigação da temática pela ótica dos profissionais.

Contribuições dos autores: Marini BPR: Concepção, estruturação metodológica, pesquisa e análise dos dados, redação e revisão final. Della Barba PCS: Concepção, orientação, redação e revisão final.

\section{REFERÊNCIAS}

1. Guralnick MJ. Family influences on Early Development: integrating the science of normative development, Risk and disability , and intervention. In: Maccartney K, Phillips D. Handbook of early childhood development. Oxford : Blackwell Publishers; 2006.

2. Serrano AM. Redes Sociais de Apoio e a sua Relevância para a Intervenção Precoce. Porto: Porto Editora; 2007. p. 110.

3. Fernandes MDA. Subsídios para a caracterização de programas de intervenção precoce implementados pelas equipas de apoios educativos na região de Trás-os-Montes [dissertação]. Porto: Universidade do Porto; 2001.

4. Muhlenhaupt M, Pizur-Barnekow K, Schefkind S, Chandler B, Harvison N. Occupational therapy contributions in early intervention: Implications for personnel preparation and interprofessional practice. Infants Young Children. 2015;28(2):123-32. DOI: 10.1097/IYC.0000000000000031

5. Dunst CJ. Familly and Community Life as the Contexts for Supporting and Strengthening Child Learning and Development. In: Eight National Congress on Early Intervention with Young Children. Aveiro, 2010.

6. McWilliam RA. Routines Based early intervention: Supporting young children and their families. Baltimore: Paul H. Brookes; 2010.

7. Mahoney G, MacDonald J. Autism, and developmental delays in young children: The Responsive Teaching curriculum for parents and professionals. Austin: PRO-ED; 2007.

8. European Agency for Development in Special Needs Eduaction. Early Childhood Intervention: progress and 
developments [cited 2016 Jan 15]. Available from: https:// www.european-agency.org/sites/default/files/earlychildhood-interventionprogress-and-developments_ECIreport-EN.pdf. A.

9. Bolsanello MA. Concepções sobre os procedimentos de intervenção e avaliação de profissionais em estimulação precoce. Educar. 2003;22:343-55. DOI: 10.1590/0104-4060.323.

10. Marini BPR, Lourenço MC, Della Barba PCS. Revisão sistemática integrativa da literatura sobre modelos e práticas de intervenção precoce no Brasil. Rev Paul Pediat. 2017;35(4):45663. DOI: 10.1590/1984-0462/;2017;35;4;00015.

11. Fernandes PRS. Diálogos sobre a Intervenção Precoce em Portugal e no Brasil: clarificação nacional e internacional sobre práticas profissionais [tese]. Braga: Instituto de Educação da Universidade do Minho; 2016.
12. Marini BPR. As práticas de Intervenção Precoce no Estado de São Paulo [dissertação]. São Carlos: Programa de Pós Graduação em Terapia Ocupacional da Universidade Federal de São Carlos; 2017.

13. Marini BPR, Lourenço MC, Della Barba PCS. A perspectiva brasileira das ações de Intervenção Precoce na infância. In: Fuertes M, et al. Teoria, práticas e investigação em intervenção precoce. Lisboa: CIED; 2018. p.27-49.

14. Bardin L. Análise de conteúdo. São Paulo: Edições 70; 2011.

15. Della Barba PCS. Intervenção precoce no Brasil e a prática dos terapeutas ocupacionais. Rev Inter Bras Ter Ocup. 2018;2(4):848-61. DOI: 10.47222/2526-3544.rbto14809

16. Constantinidis TC, Cunha AC. A formação em terapia ocupacional: entre o ideal e o real. Rev Ter Ocup Univ São Paulo. 2013; 24(2):149-54. DOI: 10.11606/issn.2238-6149.v24i2p149-154 